\title{
PREVALENCIA DE BIOTIPOS GINGIVALES SEGÚN EL SOMATOTIPO EN ESTUDIANTES DE LA UCSG SEMESTRE A-2017
} PREVALENCE OF GINGIVAL BIOTYPES ACCORDING TO SOMATOTYPE IN UCSG STUDENTS' SEMESTER A-2017 PREVALÊNCIA DE BIOTIPOS GINGIVAIS DE ACORDO COM SOMATOTIPO EM ESTUDANTES DA UCSG SEMESTRE A-2017

\author{
CRISTINA PAULETTE PIO PROAÑO', CARLOS ANDRES GUIM MARTÍNEZ1 \\ ${ }^{1}$ Universidad Católica de Santiago de Guayaquil; Guayaquil, Ecuador
}

RESUMEN

Introducción: el biotipo gingival tiene un impacto importante en la supervivencia de los diferentes tratamientos odontológicos. Varios autores aseguran que cada biotipo gingival tiene una respuesta diferente ante fuerzas externas. Por ello debemos tener una comprensión clara del comportamiento del mismo, para que en investigaciones posteriores se pueda conocer adecuadamente su manejo. Además, para tener un diagnóstico preciso se debe de analizar también el somatotipo del sujeto, este puede ser ectomorfo, mesomorfo y endomorfo, según sus características clínicas. Objetivo: observar la relación que existe entre el biotipo gingival y el somatotipo. Metodología: el estudio analítico es de tipo transversal se realizó en 107 estudiantes de odontología de la UCSG, seleccionados al azar según los criterios de inclusión y exclusión. Se determinó el biotipo gingival mediante el método de translucidez de la sonda en los dientes 11, 12, 13. El somatotipo se lo determino mediante el método fotográfico de sheldon, con una fotografía de cuerpo entero. Resultados: fueron analizados 107 sujetos, 72 mujeres y 35 hombres. El $57 \%$ del total de los sujetos tuvieron biotipo grueso, el $43 \%$ mostró biotipo delgado. Un 39\% mostró somatotipo ectomorfo, el 38\% somatotipo mesomorfo y el 22\% somatotipo endomorfo. Al relacionar las dos variables, de somatotipo ectomorfo un $90 \%$ =biotipo delgado, $10 \%=$ biotipo grueso, de somatotipo endomorfo un $100 \%=$ biotipo grueso, de somatotipo mesomorfo un $80 \%=$ biotipo grueso y un $20 \%$ biotipo delgado. Conclusión: el somatotipo más prevalente fue ectomorfo, y el biotipo más encontrado fue el grueso. Concluyendo de esta manera que no hay una relación clara entre el somatotipo y el biotipo gingival.

PALABRAS CLAVES: encía, biotipos gingivales, somatotipos, morfología dental, tejidos blandos.

ABSTRACT

Introduction: the gingival biotype has a significant impact on the survival of the different dental treatments. Several authors assure that each gingival biotype has a different response to external forces. For this reason, we must have a clear understanding of its behavior, so that subsequent investigations, and its management, can be properly known. In addition, to have an accurate diagnosis, the subject's somatotype must also be analyzed, this can be ectomorphic, mesomorphic, and endomorphic, depending on their clinical characteristics. The objective of this research is to observe the relationship that exists between the gingival biotype and the somatotype. Methodology: the cross-sectional analytical study was carried out in 107 UCSG dental students, randomly selected according to the inclusion and exclusion criteria. The gingival biotype was determined by the probe translucency method in teeth 11, 12, 13. The somatotype was determined by the Sheldon photographic method, with a full-body photograph. Results: 107 subjects were analyzed, 72 women and $35 \mathrm{men}$. $57 \%$ of the total subjects had a thick biotype, $43 \%$ showed a thin biotype. 39\% showed ectomorphic somatotype, $38 \%$ mesomorphic somatotype, and $22 \%$ endomorphic somatotype. When relating the two variables, $90 \%$ of ectomorphic somatotype $=$ thin biotype, $10 \%=$ thick biotype, $100 \%$ of endomorphic somatotype = thick biotype, $80 \%$ of mesomorphic somatotype $=$ thick biotype and $20 \%$ thin biotype. Conclusion: the most prevalent somatotype was ectomorphic, and the most common biotype was the thick one. The study concludes that there is no clear relationship between the somatotype and the gingival biotype

KEYWORDS: gingival biotypes, somatotipes, dental morphology, soft tissues.

RESUMO

Introdução: o biótipo gengival tem importante impacto na sobrevivência dos diferentes tratamentos odontológicos. Vários autores afirmam que cada biótipo gengival tem uma resposta diferente às forças externas. Por esse motivo, devemos ter uma compreensão clara de seu comportamento, para que em investigações posteriores seu manejo seja devidamente conhecido. Além disso, para um diagnóstico preciso, também deve ser analisado o somatótipo do sujeito, que pode ser ectomórfico, mesomórfico e endomórfico, dependendo de suas características clínicas. 0 objetivo desta pesquisa é observar a relação que existe entre o biótipo gengival e 0 somatótipo. Metodologia: 0 estudo transversal analítico foi realizado em 107 estudantes de odontologia da UCSG, selecionados aleatoriamente de acordo com os critérios de inclusão e exclusão. 0 biótipo gengival foi determinado pelo método da translucidez da sonda nos dentes 11, 12, 13.0 somatótipo foi determinado pelo método fotográfico de Sheldon, com fotografia de corpo inteiro. Resultados: foram analisados 107 sujeitos, sendo 72 mulheres e 35 homens. 57\% do total de indivíduos tinham um biótipo espesso, 43\% mostraram um biótipo fino. 39\% apresentaram um somatótipo ectomórfico, 38\% um somatótipo mesomórfico e 22\% um somatótipo endomórfico. Ao relacionar as duas variáveis, 90\% do somatótipo ectomórfico = biótipo delgado, 10\% = biótipo espesso, $100 \%$ do somatótipo endomórfico = biótipo espesso, 80\% do somatótipo mesomórfico = biótipo espesso e 20\% do biótipo delgado. Conclusão: o somatótipo mais prevalente foi o ectomórfico, e o biótipo mais comum foi o espesso. Concluindo desta forma que não existe uma relação clara entre o somatótipo e 0 biótipo gengival

PALAVRAS-CHAVE: biotipos gingivais, somatotipos, morfología dentária, tecidos macios. 
INTRODUCCIÓN

En los últimos años el grosor de la encía queratinizada ha tomado más fuerza como predictor del comportamiento del periodonto ante las fuerzas externas. ${ }^{1}$

Olsson y Lindhe (1991), identificaron una variación existente entre los seres humanos con respecto a las características morfológicas del periodonto, clasificándolo según su grosor y los identificaban por dos morfotipos gingivales, denominados biotipos: delgado $(46,7 \%)$ cuando los dientes anteriores presentan una corona clínica larga, estrecha y hueso alveolar delgado. A diferencia de un biotipo grueso (53, 3\%) presenta en los dientes anteriores coronas clínicas cortas, amplias y hueso alveolar grueso. ${ }^{14}$ Sin embargo, Müller y Eger (1997) reportaron haber encontrado biotipo delgado en pacientes con dientes que tenían coronas cortas y amplias, también en dientes con coronas estrechas y largas, Figura 1: a) Ectomorfo b) Mesomorfo c) endomorfo, por lo que podría haber tres biotipos periodontales. ${ }^{12}$ En este estudio también se constató que el sexo influye mucho en las características fenotípicas de la encía. Llegando a la conclusión que la gingiva marginal es más delgada en individuos de sexo femenino que masculino (Müller 2000). ${ }^{4}$

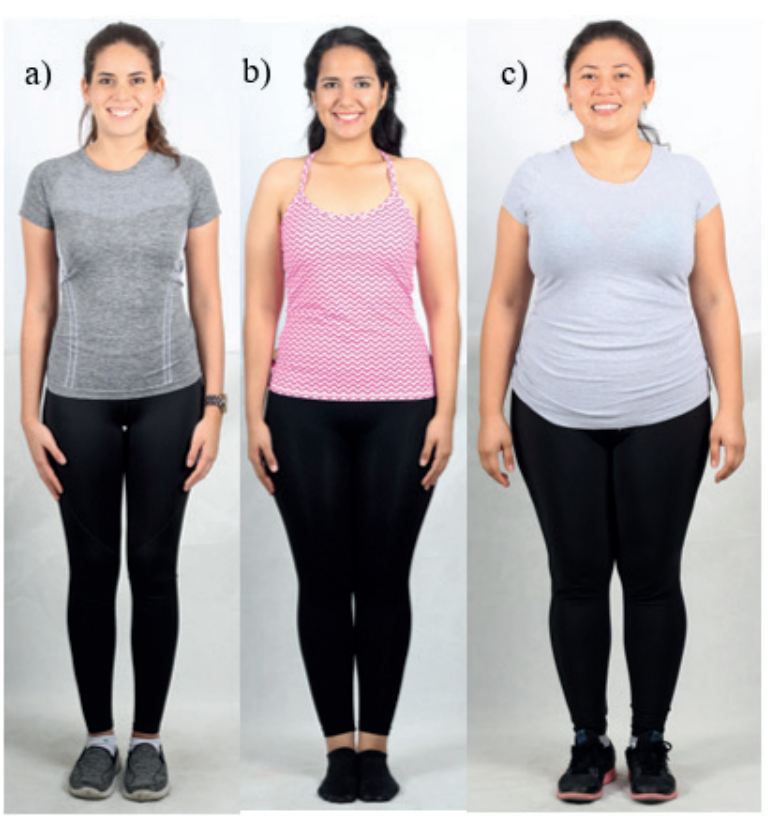

Figura 1. a) Ectomorfo b) Mesomorfo c) Endomorfo.

La apariencia clínica del periodonto marginal saludable difiere de un sujeto a otro. Muchas características clínicas se determinan genéticamente, otras están influenciadas por el tamaño, forma, posición de los dientes, y los fenómenos biológicos como el crecimiento, obesidad o el envejecimiento. ${ }^{1}$ Zeron (2011) encontró que no existe diferencias significativas entre los grupos de edad, determinando que el grosor de la encía es dado genéticamente; no varía con el crecimiento o maduración.

El análisis del somatotipo ha sido realizado en poblaciones normales de diferentes edades, sexo y niveles socioeconómicos para conocer las características biotipológicas de estos grupos humanos (Katzmarzyk et al, 1998; Katzmarzyk \& Malina, 1999). Los valores específicos de sus componentes han sido correlacionados en diferentes patologías, enfermedad periodontal y obesidad (Magnusson et al., 1998). ${ }^{6}$

Desde este punto de vista la antropometría tiene como finalidad conocer las características morfológicas de una muestra, para poder visualizar esas características se usa la técnica del somatotipo. Esta usa los componentes Endomórfico, Mesomórficos y Ectomórfico. El concepto vigente del Somatotipo fue propuesto por Heath y Carter, quienes se apoyaron en los conceptos teóricos de Sheldon (Carter \& Heath, 1990). ${ }^{30}$

La finalidad de la presente investigación es, identificar ambos biotipos gingivales, así como la relación con el sexo y la edad y el somatotipo, utilizando las técnicas de presencia o ausencia de translucidez de la sonda periodontal, ${ }^{13}$ aspecto clínico de la morfología dental, método fotográfico de Sheldon, así de esta manera poder establecer un punto de partida para que futuras investigaciones puedan evaluar la respuesta de ambos biotipos gingivales, ante situaciones clínicas que incidan en el periodonto.

\section{METODOLOGÍA}

Se realizó un estudio analítico de tipo transversal donde se examinaron 107, estudiantes de la carrera de Odontología de la Universidad Católica de Santiago de Guayaquil inscritos en el semestre A-2017, 35 hombres y 72 mujeres. La selección de los pacientes se efectuó al azar, en base a los siguientes criterios de inclusión:

- Mayores de edad

- Estudiantes de odontología en la UCSC

- Dentición permanente

- Presenciademínimoseisdientesanterosuperiores

- Salud periodontal

Los criterios de exclusión fueron:

- Estudiantes con enfermedad periodontal activa 
- Estudiantes con secuelas de enfermedad periodontal

- Menores de edad

- Estudiantes edéntulos antero superiores

- Estudiantes con ortodoncia superior

- Estudiantes con prótesis fija anterosuperiores

Todos los estudiantes firmaron un consentimiento informado, en él se explicaba el procedimiento a realizar, el cual fue aprobado por el comité deética de la Universidad Católica Santiago de Guayaquil. Para la medición del somatotipo se usó el método fotográfico de Sheldon, una fotografía de cuerpo entero tomada con una cámara Canon EOS Rebel T6 colocada entre 5 a $10 \mathrm{~m}$, la altura del lente debe ser a 1,10 m del suelo para fotografiar a adultos. La distancia focal es de $135 \mathrm{~mm}$ para $10 \mathrm{~m}$ y $50 \mathrm{~mm}$ para $4,5 \mathrm{~m} .{ }^{18}$ Fotografías intraorales con retractor de carrillos, abrebocas en oclusión con la sonda periodontal en la parte media del surco de los dientes 11, 12, 13; se utilizó una sonda carolina del norte cuya calibración es milimetrada hasta los $12015 \mathrm{~mm}$.

Para la recolección de datos se usó una ficha clínica elaborada específicamente para este estudio. Las variables medidas fueron: género, edad, somatotipo, morfología dental y biotipo periodontal. Alutilizar el método visual para el somatotipo se asignó a los pacientes las siguientes categorías descritas por William H Sheldon: somatotipo endomorfo cuando tenga predominio de desarrollo visceral; gordura; su estructura ósea y muscular está poco desarrollada y es débil.

A diferencia de cuando tenga predominio de las estructuras corporales: huesos, músculos y tejido conectivo, lo que proporciona un aspecto físico fuerte y resistente el tronco es largo y musculoso; el volumen del tórax es superior al del abdomen; la piel es gruesa será somatotipo Mesomorfo. Y en el caso de tener organismo demacrado, de músculos pobres y huesos delicados; pecho aplastado; extremidades largas y delgadas será ectomorfo.

La determinación del biotipo periodontal mediante la transparencia de la sonda se realizó mediante el sondaje de la parte media del surco gingival de los dientes 11,12 y 13 con sonda carolina del norte, estéril. La determinación del biotipo delgado se estableció al observar la transparencia de la sonda a través de la encía y biotipo grueso si no se observaba o transparentaba la sonda, según lo descrito por kan y col en el 2010. (Figura 2).

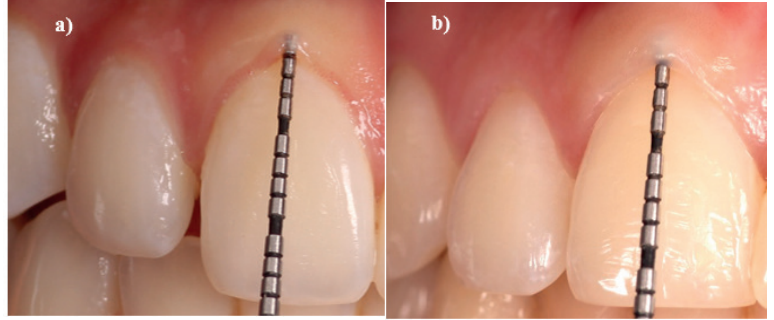

Figura 2. a) biotipo delgado, b) biotipo grueso.

Para el análisis de los datos se tabulóla información en Microsoft Excel para este análisis se utilizó el software estadístico R SPSS y Excel en la parte gráfico. Primero se realizó un análisis univariado mediante tablas de frecuencia y gráficos en barras a histogramas, seguidamente se realizó en análisis bivariado en el cual se utilizó para determinar la relación entre variables numéricas el test $\mathrm{t}$ student; y para determinar la asociación entre variables categóricas; la prueba Chi cuadrado de Pearson, que indica que se acepta la hipótesis nula si el coeficiente era inferior a 0.05 .

\section{RESULTADOS}

Participaron en el estudio 109 sujetos, 35 hombres (33\%) y 72 mujeres $(67 \%)$. La muestra fue dividida en tres subgrupos según la edad. En el grupo entre 18 y 20 años el biotipo periodontal más prevalente fue el grueso con el $64 \%$ y el delgado con $36 \%$, en el grupo de 21 - 24 un 50\% presentó biotipo grueso, al igual que el delgado con un $50 \%$, y finalmente el grupo de 25 - 30 un $100 \%$ tuvo biotipo grueso. Al evaluar el biotipo gingival el $57 \%$ de los sujetos mostró un biotipo grueso mientras que el 43\% mostró un biotipo delgado. Dando como resultado mayor prevalencia de biotipo grueso (Tabla 1). Al relacionar el biotipo gingival con el género se mostró que el más prevalente en ambos fue el biotipo grueso, el 54\% de mujeres mostraron biotipo gingival grueso, el 46\% biotipo delgado. En el género masculino un $63 \%$ mostró biotipo grueso y un $37 \%$ biotipo delgado. Al evaluar la relación entre ambos, los resultados no fueron estadísticamente significativos (test chicuadrado de Pearson= 0.394).

TABLA 1. FRECUENCIA Y PORCENTAJE DE BIOTIPOS GINGIVALES

\begin{tabular}{lrr}
\hline BIOTIPO GINGIVAL & FRECUENCIA & $\%$ \\
\hline Biotipo grueso & 61 & $57 \%$ \\
\hline Biotipo delgado & 46 & $43 \%$ \\
\hline Total & 107 & $100 \%$
\end{tabular}

Relacionado el biotipoperiodontal con la morfología dental, el biotipo grueso fue el más prevalente, el 97\% de morfología dental cuadrada mostró 
biotipo grueso y el 3\% biotipo delgado. El 90\% de morfología dental ovoide mostró biotipo grueso y el $10 \%$ biotipo delgado. Finalmente, el 14\% de morfología dental triangular mostró un biotipo grueso y el $86 \%$ delgado. Evaluando el somatotipo el $39 \%$ del total de los sujetos fueron ectomorfo, el $22 \%$ fueron endomorfo yel $38 \%$ mesomorfo (Tabla 2).

TABLA 2. FRECUENCIA Y PORCENTAJE DE SOMATIPOS

\begin{tabular}{lrr} 
SOMATIPO & FRECUENCIA & $\%$ \\
\hline Ectomorfo & 42 & $39 \%$ \\
\hline Endomorfo & 24 & $22 \%$ \\
\hline Mesomorfo & 41 & $38 \%$ \\
\hline Total & 107 & $100 \%$ SSS
\end{tabular}

Al relacionar los resultados obtenidos entre el biotipo periodontal y el somatotipo, se observó que de los sujetos que mostraron somatotipo ectomorfo fueron 42 (39\%) un $90 \%$ tuvieron un biotipo delgado y un $10 \%$ tuvieron biotipo grueso, de los que mostraron somatotipo endomorfo 24 (22\%) un $100 \%$ tuvieron biotipo grueso, y los que mostraron somatotipo mesomorfo 41 (38\%) un $80 \%$ tuvieron biotipo grueso, y un $20 \%$ biotipo delgado. (Tabla 3).

TABLA 3. RELACIÓN ENTRE BIOTIPO GINGIVAL Y SOMATIPO.

\begin{tabular}{|c|c|c|c|}
\hline \multirow[b]{2}{*}{ SOMATOTIPO } & \multicolumn{2}{|c|}{ BIOTIPO FACIAL } & \multirow{2}{*}{$\begin{array}{r}\text { TOTAL } \\
\text { GENERAL }\end{array}$} \\
\hline & $\begin{array}{l}\text { BIOTIPO } \\
\text { GRUESO }\end{array}$ & $\begin{array}{r}\text { BIOTIPO } \\
\text { DELGADO }\end{array}$ & \\
\hline Ectomorfo & 4 & 38 & 42 \\
\hline Endomorfo & 24 & & 24 \\
\hline Mesomorfo & 33 & 8 & 41 \\
\hline Total general & 61 & 46 & 107 \\
\hline \multirow[b]{2}{*}{ SOMATOTIPO } & \multicolumn{3}{|c|}{ BIOTIPO FACIAL } \\
\hline & $\begin{array}{l}\text { BIOTIPO } \\
\text { GRUESO }\end{array}$ & $\begin{array}{r}\text { BIOTIPO } \\
\text { DELGADO }\end{array}$ & $\begin{array}{r}\text { TOTAL } \\
\text { GENERAL }\end{array}$ \\
\hline Ectomorfo & $10 \%$ & $90 \%$ & 100\% \\
\hline Endomorfo & $100 \%$ & $0 \%$ & $100 \%$ \\
\hline Mesomorfo & $80 \%$ & $20 \%$ & $100 \%$ \\
\hline Total general & $57 \%$ & $43 \%$ & 100\% \\
\hline
\end{tabular}

\section{DISCUSIÓN}

La apariencia de una encía sana se refleja en la estructura subyacentedel epitelioy la lámina propia. La posición de los dientes durante la erupción da la distribución topográfica de la encía, inclusive la forma de los dientes es muy importante para dar las características clínicas de la encía. Por esto se podría decir que el biotipo gingival está influenciado tanto por factores genéticos como medioambientales. ${ }^{14}$
Se ha encontrado varios artículos donde se relaciona la edad, sexo, morfología dental, métodos para diagnosticar los biotipos gingivales mas no uno específico donde se hable de una relación directa con el somatotipo. Sin embargo, se analizó estudios previos en donde se revisan de manera individual nuestras variables.

El diagnóstico tanto del biotipo gingival como el somatotipo, dependen de múltiples parámetros para que sean confiables, es necesario más de un método para su diagnóstico. ${ }^{13,14}$

Estudios previos han mostrado ya una variación considerable entre los individuos con respecto a las características morfológicas del periodonto y de los dientes. Ya en 1989 se sugirió la existencia de distintos morfotipos los llamados "biotipos periodontales" (Seibert \& Lindhe 1989). ${ }^{13}$ Pos- $^{-}$ teriormente, las características específicas de estos biotipos fueron bien definidas por Olsson et al. (1993) 1

Olsson y Lindhe, establecen una relación entre la forma del incisivo superior y biotipo periodontal de toda la boca; ${ }^{14}$ sin embargo, en el estudio de Eger en $1996^{12}$ establece que no hay una correlación entre la forma del diente y el grosor de la encía, asegurando que el grosor se correlaciona con la amplitud de la encía queratinizada. ${ }^{12,14}$

Enesteestudio, seutilizóel métodode transparencia de la sonda descrito por Kan et al. En 2009. ${ }^{15}$ Este es un método no invasivo para diferenciar entre el biotipo grueso y delgado basado en la visibilidad de la sonda a través del margen gingival. De Rouck et al. En 2009 usó este método, mostrando el 85\% de éxito en la reproducibilidad de las mediciones en su muestra. ${ }^{13}$

Se decidió sólo incluir los incisivos centrales como dientes de referencia porque las diferencias entre los biotipos periodontales son más explícitas para estos dientes y porque sus características específicas se encuentran fácilmente a diferencia de en otros dientes (Olsson \& Lindhe 1991, Olsson et al., 1993, Mu. Et al., 2000a). En el presente estudio fue diagnosticado el biotipo gingival en todos los participantes mediante el método de translucidez de la sonda periodontal y método visual, dando como resultado mayor prevalencia de biotipo grueso con el $57 \%$ seguido del biotipo delgado con un $34 \%$ en el total de muestra. Aligual que en varios estudios no se encontró diferencia estadísticamente significativa. 
Según Kan J et al en 2009, en su estudio evaluaron el biotipo periodontal en 48 pacientes con los mismos métodos que se utilizó, y al comparar los resultados no hubieron diferencias significativas, concluyendo que ambos métodos son eficaces para determinar el biotipo periodontal. ${ }^{14,15} \mathrm{Sin}$ embargo Zweers et al en 2014 habla de que aún no hay una evidencia científica sólida para catalogar al metido visual como totalmente eficiente, concordando con lo obtenido en el estudio de Eghbali A. et al en el 2009 en el que un solo un $50 \%$ de la muestra se pudo identificar correctamente con este método, siendo el biotipo grueso el más rápido de reconocer, así como en el presente estudio. Llegando a la resolución que el método de translucidez de la sonda es usado con mayor frecuencia ya que asegura mayor precisión que el método visual, como lo indica Fischer et al 2014, comprobando que este método es eficaz para determinar el biotipo gingival. ${ }^{16}$

Abraham S. et al en 2015, tuvieron como resultado mayor prevalencia de biotipo grueso en varones y delgado en mujeres, también lograron afirmar que las personas jóvenes están más predispuestas a mostrar un biotipo grueso. Afianzando la teoría de que las coronas cortas y anchas estaban relacionadas a biotipos gruesos y por el contrario coronas largas y angostas pertenecían a un biotipo delgado. ${ }^{17}$ Una explicación de esta observación se ha proporcionado anteriormente también: los sujetos de estudio con una forma de corona cuadrática tienen un periodonto más grueso. Por el contrario, los individuos con una forma de corona cónica tienden a tener un periodonto relativamente más delgado (Weisgold 1977, Sebert \& Lindhe 1989, Olsson \& Lindhe 1991). Rathee M en 2016, inclina su investigación de acuerdo con Abraham $S$ en que el biotipo grueso se presenta con mayor frecuencia en hombres, sin embargo, en su estudio no hay un porcentaje relevante, que indique que el biotipo varía con la edad. ${ }^{8}$ Shah $\mathrm{R}$ et al en (2016) afirma que no existe ninguna relación entre el biotipo periodontal con la edad y el sexo, pero está de acuerdo al igual que todos los autores mencionados y también con el presente estudio que el biotipo de mayor prevalencia en la comunidad es el grueso.

En relación al somatotipo los participantes fueron diagnosticados bajo el método fotográfico de sheldon en donde el más común fue ectomorfo con el $39 \%$, seguido del mesoformo con $38 \%$ y al final endomorfo con el $22 \%$. Con referencia a diversas investigaciones sobre el somatotipo,
Beddury cols (2004), realizaron un estudio con 828 estudiantes universitarios en donde los resultados indicaron prevalencia de somatotipo ectomorfo en ambos sexos, por tener valores bajos en la endomorfia, importante masa osteo-muscularcon valores relativamente altos en la mesomorfia. ${ }^{19}$ Corroborando lo que indica el presente estudio, mayor prevalencia de sujetos con somatotipo ectomorfo y mesomorfo. ${ }^{18,19}$ Galic en 2016 al igual que Beddur en su estudio constató que el somatotipo mesomorfo fue el más prevalente en su estudio. ${ }^{31}$

\section{CONCLUSIÓN}

Si bien es cierto que existieron limitaciones de número de sujetos en el presente estudio y homogeniedad de la muestra, se logró concluir que: el biotipo grueso fue es más prevalente en toda la muestra con un $57 \%$, no existiendo diferencias significativas entre hombres y mujeres, y está presente en mayor porcentaje en el somatotipo endomorfo con un $(100 \%)$, seguido por el somatotipo mesomorfo con un $(80 \%)$, y en menos porcentaje por el somatotipo ectomorfo con el $(10 \%)$. El somatotipo más encontrado en el grupo de estudio fue el ectomorfo (39\%), seguido por el somatotipo mesomorfo (38\%) y por último somatotipo endomorfo (22\%), concluyendo de esta manera que no hay una relación clara entre el somatotipo y el biotipo gingival sólo representa una característica clínica de estructuras y funciones celulares. Existen muchos métodos para determinar el biotipo gingival como el visual y la transparencia de la sonda periodontal. Sin embargo, el método visual no puede ser el único método que se use para determinar el biotipo gingival por su falta de tecnicismo en el mismo. Siendo la transparencia de la soda el método más certero.

Según la edad no se encontró ninguna variación en el biotipo gingival, indicándonos que dicha característica se da durante el proceso de consolidación de la dentición permanente. Al igual que en la morfología dental, se establece que no hay una relación directa entre el biotipo gingival y el radio del incisivo superior, indicándonos que además de la forma del órgano dental existen otros factores importantes para el diagnóstico del biotipo gingival.

Se llegó a la conclusión, que, como odontólogos, no solo debemos fijarnos en la parte dental sino también en todas las características físicas del paciente. Lo recomendable es hacer un análisis 
antropométrico para encontrar la mejor definición de los diferentes biotipos del paciente. Va a ser de vital importancia saber reconocer al biotipo gingival y todas sus características, y así poder evitar fracasos en tratamientos por un mal diagnóstico del mismo.

Son necesarias más investigaciones que evalúen los métodos utilizados aumentando el tamaño muestral, como son los estudios de prevalencia y ensayos clínicos controlados, donde las variables son estrictamente controladas.

\section{REFERENCIAS BIBLIOGRÁFICAS}

1. Zerón A, Biotipos, fenotipos y genotipos. ¿Qué biotipo tenemos? (Segunda parte) Revista Mexicana de Periodontología 2011; 2(1): 22-33 23

2. Botero $P$, evaluación de los biotipos en dentición permanente, rev. CES odontologia, vol 14, no 2,2001

3. Navarrete $M$, Correlación entre biotipo gingival, ancho y grosor de encía adherida en zona estética del maxilar superior, Rev Clin Periodoncia Implantol Rehabil Oral. 2015;8(3):192-197

4. Rezaei Z, Gingival biotype: a review, Rev. General Dentistry, July

5. Shah R, Sowmya NK, Thomas R, Mehta DS. Periodontal biotype: Basics and clinical considerations. J Interdiscip Dentistry 2016; 6:44-9.

6. Soca P, consecuencias dela obesidad, ACIMED. 2009; 20(4): 84-92

7. K. R. Nagaraj, Ravindra C. Savadi, Anupama R. Savadi, Gingival Biotype - Prosthodontic Perspective, Journal of Indian Prosthodontic Society (March 2010) 10:27-30

8. Rathee M, Rao PL, Bhoria M. Prevalence of Gingival Biotypes among Young Dentate North Indian Population: A Biometric Approach. Int J Clin Pediatr Dent 2016;9(2):104-108.

9. R. G. Shiva Manjunath et al., Gingival Biotype Assessment in a Healthy Periodontium: Transgingival Probing Method, Journal of Clinical and Diagnostic Research. 2015 May, Vol-9(5): ZC66-ZC69 67

10. Memon, etal.: Assessing the gingival biotype for the reliability by three different methods, The Journal of Indian Prosthodontic Society | Oct-Dec 2015 | Vol 15 | Issue 4

11. Cuny-Houchmand et al. Gingival Biotype Assessement: Visual Inspection Relevance And Maxillary Versus Mandibular Comparison, The Open Dentistry Journal, 2013, 7, 1-6
12. Müller H, Eger T, Gingival phenotypes in young male adults. J Clin Periodontal 1997; 24: 65-71

13. De Rouck T, Eghbali R, Collys K, De Bruyn $\mathrm{H}$, Cosyn J. The gingival biotype revisited: transparency of the periodontal probe through the gingival margin as a method to discriminate thin from thick gingiva. J Clin Periodontol 2009; 36: 428-433. doi: 10.1111/j.1600-051X.2009.01398.x.

14. Olsson M and Lindhe J: Periodontal characteristics in individuals with varying form of the upper central incisors. J Clin Periodontol 1991; 18: 78-82.

15. Kan J, Morimoto T, Rungcharassaeng K, Roe P, Smith D. Gingival Biotype assessment in the esthetic zone: visual versus direct measurement. Int Periodontics Restorative Dent. 2010; 30:237--242.

16. Fischer K, Richter T, Kebschull M, Petersen $\mathrm{N}$, Fickl S, On the relationship between gingival biotypes and gingival thickness in young Caucasians. Clinical Oral Implants Research. 2014; 26(8):865-869.

17. Abraham S, Deepak K, Ambili R, Preeja C, Archana V. Cingival biotype and its clinical significance - A review. The Saudi Journal for Dental Research. 2014; 5(1):3-7.

18. Garrido R, Gonzalez M, Vercher M. Correlación entre los componentes del somatotipo y la composición corporal según formulas antropométricas. Revista Digital - Buenos Aires - Año 10 - Nº 84 - Mayo de 2005

19. AguilarY, CastellanosR, CobosA. Características somatotípicas de una muestrade estudiantes de educación universitaria. Revista Digital. Buenos Aires, Año 16, № 160, Septiembre de 2011.

20. Martinez C.; Silva H.; Collipal E. \& Carrasco V. Descripcióndel somatotipoeIMCenuna muestra de Adolescentes de colegios municipalizados de la ciudad de Temuco- Chile. Int. J. Morphol., 26(3):653-657, 2008.

21. Castellanos et al. / Estudio del somatotipo como posible parámetro de enfermedades en estudiantes de Odontología, multiciencias VOL. 10, No Extraordinario, 2010 (265 - 270)

22. Arce P, Flores A, Lelievre C, Changes of somatotype in high school students, $V$ region, Chile: 1985-2010, Nutr Hosp. 2012;27(1):270-275

23. Lizana P, Olivares R, Berral F. Somatotype tendency in Chilean adolescents from Valparaíso: review from 1979 to 2011, Nutr Hosp. 2015;31(3):1034-1043 
24. Silva, H., Bruneau, J. C., Reyno, H. P., \& Bucarey, S.. (2003). Somatotype and body mass index from a both sexes adolescent's sample in temuco, chile. International Journal of Morphology, 21(4), 309-313.

25. Zweers J, Thomas R, Slot D, Weisgold A, Van der Weijden F. Characteristics of periodontal biotype, its dimensions, associations and prevalence: a systematic review. Journal of Clinical Periodontology. 2014; 41(10):958-971

26. Abraham S, Athira S. Correlation of Gingival Tissue Biotypes with Age, Gender and Tooth Morphology: A Cross Sectional Study Using Probe Transparency Method. Journal of Dental and Medical Sciences. 2015; 14(9):64- 69.

27. Geron S, Atalia W. Influence of sex on the perception of oral and smile esthetics with different gingival display and incisal plane inclination. Angle Orthod 2005; 75(5): 778-784.

28. Bhat V, Shetty S. Prevalence of different gingival biotypes in individuals with varying forms of maxillary central incisors: A survey. Journal of Dental implants. 2013; 3(2):116.

29. Ahmad I. Anterior dental aesthetics: Gingival perspective. British Dental Journal. 2005;199(4):195-202.

30. Fu J, Lee A, Wang HL. Influence of tissue biotype on implant esthetics. Int J Oral Maxillofac Implants

31. Galic B, Pavlica P, Mirjana U, et al. Somatotype characteristics of normal-weight and obese women among different metabolic subtypes, Arch Endocrinol Metab. 2016;60. 